\title{
Stabilized scattering wave-function calculations using the Lippmann-Schwinger equation for long conductor systems
}

\author{
Shigeru Tsukamoto, ${ }^{1, *}$ Yoshiyuki Egami, ${ }^{2}$ Kikuji Hirose, ${ }^{3}$ and Stefan Blügel ${ }^{1}$ \\ ${ }^{1}$ Peter Grünberg Institute and Institute for Advanced Simulation, Forschungszentrum Jülich and JARA, D-52425 Jülich, Germany \\ ${ }^{2}$ Nagasaki Advanced Computing Center, Nagasaki University, Nagasaki 852-8521, Japan \\ ${ }^{3}$ Department of Precision Science \& Technology and Applied Physics, Osaka University, Suita, Osaka 565-0871, Japan
}

(Received 11 July 2011; published 23 September 2011)

\begin{abstract}
We present an improvement of the Lippmann-Schwinger equation method, which calculates electron-scattering wave functions of a nanoscale conductor suspended between a pair of electrodes. The improvement eliminates the numerical collapse which frequently occurs while solving the Lippmann-Schwinger equation for long conductor systems and originates from evanescent wave components of the retarded Green's function of the LippmannSchwinger equation. We introduce regularization and ratio expression into the Green's function matrix and discover that the resultant Green's function does not suffer from the numerical collapse without increasing computational cost. As a performance test, we carry out electron transport calculations of Al monoatomic linear chains with a length of up to 75.6 bohrs. The numerical test demonstrates that the improved Lippmann-Schwinger equation method is applicable to long conductor systems with no numerical collapse and adequate computational accuracy.
\end{abstract}

DOI: 10.1103/PhysRevB.84.115443

PACS number(s): 73.63.-b, 31.15.-p, 62.23.Hj

\section{INTRODUCTION}

In view of the recent progress in nanostructure fabricating technology, electron transport properties of nanoscale functional systems have attracted a great deal of attention over the years, because electron transport properties are recognized as one of the critical issues for developing nanoscale functional electronic devices in the near future. So far, a number of studies have been conducted experimentally and theoretically for electron transport through nanostructures, e.g., tunneling junctions, quantum point contacts, atomic chains, and organic molecules suspended between a pair of electrodes. ${ }^{1}$ In the field of theoretical study, various types of calculation methods have been developed to simulate scattering wave functions inside nanostructures and to investigate their electron transport properties, including the nonequilibrium Green's function method, ${ }^{2}$ the recursion transfer matrix method, ${ }^{3}$ the overbridging boundary matching method, ${ }^{4}$ and the LippmannSchwinger equation method. ${ }^{5-7}$

The Lippmann-Schwinger equation method, which we are focusing on here, was first developed by Lang and Williams and applied to a study of atomic chemisorption on a metal surface. ${ }^{8}$ This method solves the integral equation of the second-kind Fredholm type with respect to an unknown scattering wave function $\psi(\boldsymbol{r})$ under an effective potential $V_{\text {eff }}(\boldsymbol{r})$, referred to as the Lippmann-Schwinger equation:

$$
\begin{gathered}
\psi(\boldsymbol{r})=\phi_{\mathrm{ref}}(\boldsymbol{r})+\int \mathcal{G}\left(\boldsymbol{r}, \boldsymbol{r}^{\prime}\right) V_{\mathrm{dif}}\left(\boldsymbol{r}^{\prime}\right) \psi\left(\boldsymbol{r}^{\prime}\right) d \boldsymbol{r}^{\prime}, \\
V_{\mathrm{dif}}(\boldsymbol{r})=V_{\mathrm{eff}}(\boldsymbol{r})-V_{\mathrm{ref}}(\boldsymbol{r}) .
\end{gathered}
$$

Here $\phi_{\text {ref }}(\boldsymbol{r}), V_{\text {ref }}(\boldsymbol{r})$, and $\mathcal{G}\left(\boldsymbol{r}, \boldsymbol{r}^{\prime}\right)$ are the scattering wave function, potential, and retarded Green's function of a reference system, respectively. After several improvements, this is now considered to be an accurate and rapid method for computing scattering wave functions of a nanostructure between a pair of electrodes and has been used for a number of investigations on electron transport properties. ${ }^{9-13}$ One of the improvements is that by keeping the reference potential $V_{\text {ref }}(\boldsymbol{r})$ constant over the plane perpendicular to the direction of electron flow, the three-dimensional real-space representation of the Lippmann-Schwinger equation (1) can be transformed into the Laue representation, in which functions are expanded using plane waves only in the directions perpendicular to electron flow. Moreover, the resultant retarded Green's function $\mathcal{G}\left(\boldsymbol{r}, \boldsymbol{r}^{\prime}\right)$ can be written in a variable-separable form. On the basis of this improvement, computational costs for numerical integration in the Lippmann-Schwinger equation (1), which is the most expensive part in terms of both memory consumption and executing time, can be drastically reduced. The other improvement is that the Green's function in the Laue representation can be obtained analytically if the reference potential $V_{\text {ref }}(\boldsymbol{r})$ is selected as a simple function along the direction of electron flow, e.g., a constant or rectangular function. ${ }^{14}$ In practical simulations of electron transport through nanoscale conductors, rectangular reference potential is certainly appropriate, since rectangular reference potential obviously approximates a pair of bare electrodes without any nanostructures between them. Indeed, rectangular reference potential works successfully in several electron transport calculations. ${ }^{5,9-12}$

When rectangular reference potential is adopted for a long conductor system such that a long nanowire or a large molecule is sandwiched between a pair of electrodes, one may encounter the numerical difficulty that a component that is a part of the Green's function in the variable-separable form diverges in an exponential manner due to the appearance of evanescent waves. This is obviously critical in practical computation using the Lippmann-Schwinger equation method. Therefore, to simulate electron transport properties of more realistic large-scale systems, it is important to find an alternative solution or an improvement to avoid the numerical collapse.

In this paper, we propose the procedure of regularization and ratio expression for the Green's function matrix elements as a solution for avoiding the numerical collapse. The introduction of the regularization and ratio expression successfully 
contributes numerical stability to practical computation while avoiding an increase in computational cost, and we have confirmed reasonable electron transport property even for a long Al monoatomic chain with the length of $75.6 \mathrm{bohrs}$ without any numerical collapse.

This paper is organized as follows: Sec. II starts with a concise description of the Lippmann-Schwinger equation method. Then the variable-separable form of the Green's function and the numerical collapse incidental to the separable form are stated. Next, we introduce a regularization and ratio expression into the Green's function matrix. In Sec. III we demonstrate the numerical stability of the LippmannSchwinger equation method adopting the regularization and ratio expression. Throughout this paper, we use the bohr unit for length $(1 \mathrm{bohr}=0.529 \AA)$, Ry unit for cutoff energy $(1 \mathrm{Ry}=13.6 \mathrm{eV})$, and hartree unit for energy other than cutoff energy $(1$ hartree $=27.2 \mathrm{eV})$.

\section{LIPPMANN-SCHWINGER EQUATION METHOD}

A Schrödinger-like equation under an effective oneelectron potential $V_{\text {eff }}(\boldsymbol{r})$ with respect to a scattering wave function $\psi(\boldsymbol{r})$ is transformed into a Lippmann-Schwinger Eq. (1), which includes a retarded Green's function $\mathcal{G}\left(\boldsymbol{r}, \boldsymbol{r}^{\prime}\right){ }^{5,8}$ The equation describes the notion that electrons in a reference system are elastically scattered by the potential difference $V_{\text {dif }}(\boldsymbol{r})$. For the determination of scattering wave functions of a system using the Lippman-Schwinger equation method, two different types of boundary condition have been conventionally applied to a computational supercell, i.e., periodic boundary conditions in the $x$ and $y$ directions and an open boundary condition in the $z$ direction normal to the electrode surface, as shown in Fig. 1. In the simplest implementation, a part deep in each semi-infinite electrode is approximated by a jellium model ${ }^{15}$ (solid parts in Fig. 1), which has a uniform background positive charge distribution and hence a constant potential. ${ }^{16}$ The atomic structure connecting the jellium parts is treated as a perturbation, and the jellium system is then regarded as a reference system. This treatment results in the reference potential $V_{\text {ref }}(\boldsymbol{r})$ being constant over the $x$ - $y$ plane as $V_{\text {ref }}(\boldsymbol{r})=V_{\text {ref }}(z)$. Owing to the one-dimensional reference potential, the Lippmann-Schwinger equation expressed in the three-dimensional real-space representation Eq. (1) can be transformed into the Laue representation, which employs a two-dimensional plane-wave expansion in the $x$ and $y$ directions and a real-space expression in the $z$ direction, i.e.,

$$
\begin{gathered}
\psi^{(l)}(z)=\phi_{\mathrm{ref}}^{(l)}(z)+S \int \mathcal{G}^{(l)}\left(z, z^{\prime}\right) \sum_{l^{\prime}} V_{\mathrm{dif}}^{\left(l-l^{\prime}\right)}\left(z^{\prime}\right) \psi^{\left(l^{\prime}\right)}\left(z^{\prime}\right) d z^{\prime} \\
V_{\mathrm{dif}}^{(l)}(z)=V_{\mathrm{eff}}^{(l)}(z)-V_{\mathrm{ref}}(z) \delta_{l, 0}
\end{gathered}
$$

where $l$ is the index of a plane-wave expansion component, and $S$ is the cross section of the supercell in the $x$ and $y$ directions. $\psi^{(l)}(z), \phi_{\text {ref }}^{(l)}(z), \mathcal{G}^{(l)}\left(z, z^{\prime}\right)$, and $V_{\text {dif }}^{(l)}(z)$ are the plane-wave expansion coefficients of unknown scattering wave function, reference wave function, retarded Green's function, and potential difference in the Laue representation, respectively. The reference wave function is obtained by

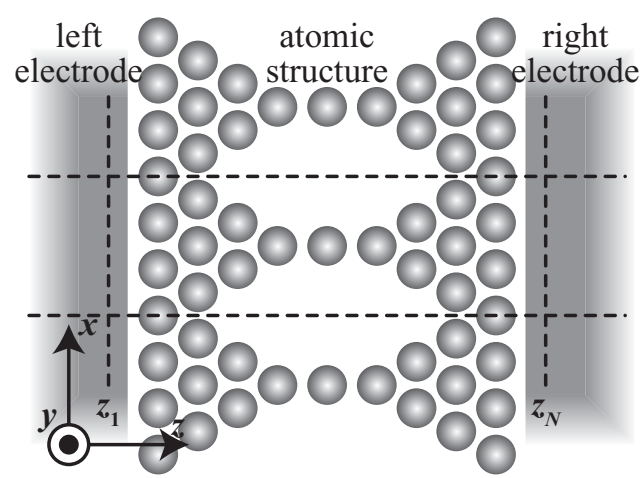

FIG. 1. Schematic representation of a conventional system that has been frequently used in electron transport calculations. An atomic structure is sandwiched between a pair of bare electrodes of a jellium model. The periodic boundary condition is applied in the $x$ and $y$ directions (horizontal dashed lines), and the open boundary condition is in the $z$ direction (vertical dashed lines).

solving a one-dimensional Schrödinger-like equation under the reference potential $V_{\text {ref }}(z)$ as

$$
\begin{gathered}
{\left[\Delta_{z}^{(l)}+V_{\mathrm{ref}}(z)\right] \phi_{\mathrm{ref}}^{(l)}(z)=E \phi_{\mathrm{ref}}^{(l)}(z),} \\
\Delta_{z}^{(l)}=-\frac{1}{2} \frac{d^{2}}{d z^{2}}+\frac{1}{2}\left|\boldsymbol{G}^{(l)}\right|^{2},
\end{gathered}
$$

where $\boldsymbol{G}^{(l)}$ is the two-dimensional wave-number vector of the $l$ th plane-wave expansion component, and $E$ is the energy of electrons injected into the system through either of the supercell boundary planes at $z=z_{1}$ and $z=z_{N}$, which have an open boundary condition. For simplicity of description, the two-dimensional Bloch wave vector is not written explicitly.

The retarded Green's function in the Laue representation, $\mathcal{G}^{(l)}\left(z, z^{\prime}\right)$, also satisfies a one-dimensional differential equation under the reference potential $V_{\text {ref }}(z)$ :

$$
\left[E-\left(\Delta_{z}^{(l)}+V_{\text {ref }}(z)\right)\right] \mathcal{G}^{(l)}\left(z, z^{\prime}\right)=\frac{1}{S} \delta\left(z-z^{\prime}\right) .
$$

Note that when reference potential $V_{\text {ref }}(z)$ is chosen so as to be the same as the effective potential $V_{\mathrm{eff}}^{(l)}(z)$ outside the supercell boundary planes at $z=z_{1}$ and $z=z_{N}$, potential difference $V_{\text {dif }}^{(l)}(z)$ is zero outside the boundary planes $\left(z<z_{1}\right.$ and $z_{N}<z$ ). Therefore, the integration range in Eq. (2) becomes finite as the interval $\left[z_{1}, z_{N}\right]$.

\section{A. Separable form of the Green's function and numerical collapse}

The retarded Green's function in the Laue representation, $\mathcal{G}^{(l)}\left(z, z^{\prime}\right)$, is known to be written in a variable-separable form ${ }^{5,10}$ i.e., each plane-wave expansion component of the Green's function is described as a product of a pair of one-dimensional scattering wave functions $y_{+}^{(l)}(z)$ and $y_{-}^{(l)}(z)$, which are injected into the reference system from deep in the left and right electrodes, respectively, as follows:

$$
\mathcal{G}^{(l)}\left(z, z^{\prime}\right)=\frac{1}{S} \frac{2}{W^{(l)}}\left\{\begin{array}{ll}
y_{-}^{(l)}(z) y_{+}^{(l)}\left(z^{\prime}\right), & z<z^{\prime} \\
y_{-}^{(l)}\left(z^{\prime}\right) y_{+}^{(l)}(z), & z^{\prime}<z
\end{array} .\right.
$$


Here $W^{(l)}$ is Wronskian being independent of the $z$ coordinate and is defined by

$$
W^{(l)}=\left|\begin{array}{cc}
y_{-}^{(l)}(z) & y_{+}^{(l)}(z) \\
\frac{d}{d z} y_{-}^{(l)}(z) & \frac{d}{d z} y_{+}^{(l)}(z)
\end{array}\right| .
$$

The Green's function components $y_{ \pm}^{(l)}(z)$ satisfy the homogeneous differential equation (3) as

$$
\left[\Delta_{z}^{(l)}+V_{\mathrm{ref}}(z)-E\right] y_{ \pm}^{(l)}(z)=0 .
$$

Since reference potential $V_{\text {ref }}(z)$ is assumed to be constant on the supercell boundary planes at $z=z_{1}$ and $z=z_{N}$, the boundary conditions of the solution $y_{ \pm}^{(l)}(z)$ can be described as linear combinations of injected, backscattered, and transmitted plane waves. Therefore, the homogeneous differential equation (7) is solved under the boundary conditions

$$
y_{+}^{(l)}(z)=\left\{\begin{array}{cc}
e^{i k_{1}^{(l)} z}+r_{+}^{(l)} e^{-i k_{1}^{(l)} z} & z<z_{1} \\
t_{+}^{(l)} e^{i k_{N}^{(l)} z} & z_{N}<z
\end{array}\right.
$$

and

$$
y_{-}^{(l)}(z)=\left\{\begin{array}{cc}
t_{-}^{(l)} e^{-i k_{1}^{(l)} z} & z<z_{1} \\
e^{-i k_{N}^{(l)} z}+r_{-}^{(l)} e^{i k_{N}^{(l)} z} & z_{N}<z
\end{array} .\right.
$$

Here $k_{1(N)}^{(l)}$ is the wave number along the $z$ direction at the outside of the boundary plane $z<z_{1} \quad\left(z_{N}<z\right)$, and $k_{1(N)}^{(l)}=\sqrt{2\left[E-V_{\text {ref }}\left(z_{1(N)}\right)\right]-\left|\boldsymbol{G}^{(l)}\right|^{2}}$. The coefficients $r_{ \pm}^{(l)}$ and $t_{ \pm}^{(l)}$ are the reflection and transmission coefficients for the reference potential, respectively, and $\left|r_{ \pm}^{(l)}\right|^{2}+\left|t_{ \pm}^{(l)}\right|^{2}=1$ is satisfied.

It is clear that the variable-separable form of the retarded Green's function is highly advantageous for numerical integration of the Lippmann-Schwinger equation (2), because either $y_{+}^{(l)}(z)$ or $y_{-}^{(l)}(z)$ as a function of the variable $z$ can be separated from the Lippmann-Schwinger integration over the variable $z^{\prime}$, and the integration no longer depends on the variable $z$. In numerical computation, the real-space $z$ axis is divided into fine grids $z_{j}$ for discretization, and hence, the Green's function in the separable form shown in Eq. (5) can be rewritten in a matrix form:

$$
\hat{\mathcal{G}}^{(l)}=\frac{1}{S} \frac{2}{W^{(l)}}\left(\begin{array}{cccc}
y_{-, 1}^{(l)} y_{+, 1}^{(l)} & y_{-, 1}^{(l)} y_{+, 2}^{(l)} & \cdots & y_{-, 1}^{(l)} y_{+, N}^{(l)} \\
y_{-, 1}^{(l)} y_{+, 2}^{(l)} & y_{-, 2}^{(l)} y_{+, 2}^{(l)} & \cdots & y_{-, 2}^{(l)} y_{+, N}^{(l)} \\
\vdots & \vdots & \ddots & \vdots \\
y_{-, 1}^{(l)} y_{+, N}^{(l)} & y_{-, 2}^{(l)} y_{+, N}^{(l)} & \cdots & y_{-, N}^{(l)} y_{+, N}^{(l)}
\end{array}\right),
$$

where $y_{ \pm, j}^{(l)} \equiv y_{ \pm}^{(l)}\left(z_{j}\right)$. To solve the simultaneous linear equations derived from the Lippmann-Schwinger equation (2), conventional conjugate gradient solvers are not applicable since the Green's function matrix is not Hermitian but symmetric. However, some types of the bi-conjugated gradient method (BiCG) are still available. ${ }^{17}$

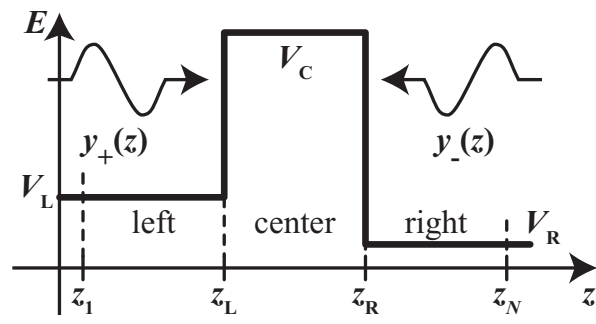

FIG. 2. An example of rectangular-shaped reference potential. The $z$ space is divided into three regions: left, center, and right regions, which correspond to $z<z_{\mathrm{L}}, z_{\mathrm{L}}<z<z_{\mathrm{R}}$, and $z_{\mathrm{R}}<z$, respectively.

We introduce a simple rectangular function, as shown in Fig. 2, for the one-dimensional reference potential $V_{\text {ref }}(z)$ :

$$
V_{\mathrm{ref}}(z)=\left\{\begin{array}{cc}
V_{\mathrm{L}} & z<z_{\mathrm{L}} \\
V_{\mathrm{C}} & z_{\mathrm{L}}<z<z_{\mathrm{R}} \\
V_{\mathrm{R}} & z_{\mathrm{R}}<z
\end{array}\right.
$$

The introduction of the simple-shaped reference potential offers several advantages for practical computation: One is that the Green's function components $y_{ \pm}^{(l)}(z)$ are analytically determined as described in the Appendix. The other is that the rectangular reference potential tends to give relatively faster convergence in a BiCG procedure when solving the Lippmann-Schwinger equation as a set of simultaneous linear equations. ${ }^{18}$ Owing to the analytical form of the Green's function components $y_{ \pm}^{(l)}(z)$, the Wronskian $W^{(l)}$ given by Eq. (6) can be represented in an analytical form as

$$
W^{(l)}=\frac{8 i k_{\mathrm{L}}^{(l)} k_{\mathrm{C}}^{(l)} k_{\mathrm{R}}^{(l)} e^{i k_{\mathrm{L}}^{(l)} z_{\mathrm{L}}} e^{-i k_{\mathrm{R}}^{(l)} z_{\mathrm{R}}} e^{i k_{\mathrm{C}}^{(l)}\left(z_{\mathrm{R}}-z_{\mathrm{L}}\right)}}{W_{0}^{(l)}}
$$

with

$$
\begin{aligned}
W_{0}^{(l)}= & \left(k_{\mathrm{C}}^{(l)}+k_{\mathrm{L}}^{(l)}\right)\left(k_{\mathrm{C}}^{(l)}+k_{\mathrm{R}}^{(l)}\right) \\
& -\left(k_{\mathrm{C}}^{(l)}-k_{\mathrm{L}}^{(l)}\right)\left(k_{\mathrm{C}}^{(l)}-k_{\mathrm{R}}^{(l)}\right) e^{2 i k_{\mathrm{C}}^{(l)}\left(z_{\mathrm{R}}-z_{\mathrm{L}}\right),}
\end{aligned}
$$

where $k_{\mathrm{L}}^{(l)}, k_{\mathrm{C}}^{(l)}$, and $k_{\mathrm{R}}^{(l)}$ are the wave numbers along the $z$ direction in the left, center, and right regions of the rectangular reference potential shown in Fig. 2, respectively. The wave numbers are defined by

$$
k_{\mathrm{L}(\mathrm{C}, \mathrm{R})}^{(l)}=\sqrt{2\left[E-V_{\mathrm{L}(\mathrm{C}, \mathrm{R})}\right]-\left|\boldsymbol{G}^{(l)}\right|^{2}} .
$$

Note that when $2\left[E-V_{\mathrm{L}(\mathrm{C}, \mathrm{R})}\right]-\left|\boldsymbol{G}^{(l)}\right|^{2}<0$, the wave number $k_{\mathrm{L}(\mathrm{C}, \mathrm{R})}^{(l)}$ can be analytically continued to an imaginary number as $k_{\mathrm{L}(\mathrm{C}, \mathrm{R})}^{(l)}=i \kappa_{\mathrm{L}(\mathrm{C}, \mathrm{R})}^{(l)}$, where

$$
\kappa_{\mathrm{L}(\mathrm{C}, \mathrm{R})}^{(l)}=\sqrt{\left|\boldsymbol{G}^{(l)}\right|^{2}-2\left[E-V_{\mathrm{L}(\mathrm{C}, \mathrm{R})}\right]} .
$$

The introduction of the separable form of the Green's function succeeded in drastically reducing computational costs, ${ }^{19}$ but it also raises the possibility of a numerical collapse that frequently occurs for long conductor systems. The numerical collapse occurs in the prefactor of the Green's function in 
the separable form Eq. (10), in which the Wronskian $W^{(l)}$ appears as the denominator. When one treats a system with a large separation between electrodes, the width of a rectangular reference potential $z_{\mathrm{R}}-z_{\mathrm{L}}$ becomes large. Moreover, when one takes the length of the two-dimensional wave-number vector $\left|\boldsymbol{G}^{(l)}\right|$ large enough to retain the accuracy in plane-wave expansion, the wave number in the $z$ direction at the center region, $k_{\mathrm{C}}^{(l)}$, becomes a large imaginary number, as seen in Eq. (15). According to the definition of the Wronskian equation (12), the numerator goes to zero in an exponential manner, while the denominator remains at a finite value of $\left(k_{\mathrm{C}}+k_{\mathrm{L}}\right)\left(k_{\mathrm{C}}+k_{\mathrm{R}}\right)$ as seen in Eq. (13). Hence, as system length increases, the Wronskian $W^{(l)}$ with a high-order plane-wave expansion goes to zero, and division by zero occurs in the computation of the prefactor of the Green's function Eq. (10). ${ }^{20}$ Furthermore, when the system size is large as $z_{1} \ll-1$ or $z_{N} \gg 1$, the Green's function components $y_{ \pm}^{(l)}(z)$ retain the potential to diverge exponentially according to their definitions Eqs. (A1) and (A2). Therefore, with respect to using the conventional separable form, sufficient cutoff energy in the plane-wave expansion would not be available when treating long conductor systems.

\section{B. Regularization and ratio expression of the Green's function matrix elements}

To avoid the above-mentioned numerical collapse in the practical computation of electron-scattering wave functions using the Lippmann-Schwinger equation method, we here introduce regularization and ratio expression into the Green's function matrix. Except where it is important, we will not explicitly write the index of the plane-wave expansion components, $l$, in the following in order to avoid complication.

So that the Wronskian $W$ causing the numerical collapse does not explicitly appear in the Green's function matrix, we regularize the diagonal elements and describe the nondiagonal elements by the ratio expression. If two quantities, $D_{j}$ and $R_{ \pm, j}$ are introduced, defined by

$$
\begin{gathered}
D_{j} \equiv \frac{2}{W} y_{-, j} y_{+, j}, \\
R_{+, j} \equiv \frac{y_{+, j}}{y_{+, j-1}}, \\
R_{-, j} \equiv \frac{y_{-, j}}{y_{-, j+1}},
\end{gathered}
$$

the Green's function matrix Eq. (10) can be rewritten as

$$
\hat{\mathcal{G}}=\frac{1}{S}\left(\begin{array}{ccccc}
D_{1} & D_{2} R_{-, 1} & D_{3} R_{-, 2} R_{-, 1} & \cdots & D_{N} \prod_{j=N-1}^{1} R_{-, j} \\
D_{1} R_{+, 2} & D_{2} & D_{3} R_{-, 2} & \cdots & D_{N} \prod_{j=N-1}^{2} R_{-, j} \\
D_{1} R_{+, 2} R_{+, 3} & D_{2} R_{+, 3} & D_{3} & \cdots & D_{N} \prod_{j=N-1}^{3} R_{-, j} \\
\vdots & \vdots & \vdots & \ddots & \vdots \\
D_{1} \prod_{j=2}^{N} R_{+, j} & D_{2} \prod_{j=3}^{N} R_{+, j} & D_{3} \prod_{j=4}^{N} R_{+, j} & \cdots & D_{N}
\end{array}\right) .
$$

Note that the matrix elements in Eq. (17) are no longer in a separable form. However, computational cost for the matrix-vector product operation is the same as the case of the variable-separable form, as mentioned later.

The diagonal elements of the Green's function matrix, $D_{j}$, which are defined by $y_{ \pm, j}$ and $W$ as Eq. (16), are analytically rendered by

$$
D_{j}= \begin{cases}\frac{1}{i k_{\mathrm{L}}}\left\{1+\left[\frac{2 k_{\mathrm{L}}\left(k_{\mathrm{C}}+k_{\mathrm{R}}\right)}{W_{0}}+\frac{2 k_{\mathrm{L}}\left(k_{\mathrm{C}}-k_{\mathrm{R}}\right)}{W_{0}} e^{2 i k_{\mathrm{C}}\left(z_{\mathrm{R}}-z_{\mathrm{L}}\right)}-1\right] e^{2 i k_{\mathrm{L}}\left(z_{\mathrm{L}}-z_{j}\right)}\right\} & z_{j}<z_{\mathrm{L}} \\ \frac{1}{i k_{\mathrm{C}}}\left[\frac{\left(k_{\mathrm{C}}+k_{\mathrm{L}}\right)\left(k_{\mathrm{C}}+k_{\mathrm{R}}\right)}{W_{0}}+\frac{\left(k_{\mathrm{C}}-k_{\mathrm{L}}\right)\left(k_{\mathrm{C}}-k_{\mathrm{R}}\right)}{W_{0}} e^{2 i k_{\mathrm{C}}\left(z_{\mathrm{R}}-z_{\mathrm{L}}\right)}\right. & z_{\mathrm{L}}<z_{j}<z_{\mathrm{R}} \\ \left.+\frac{\left(k_{\mathrm{C}}+k_{\mathrm{L}}\right)\left(k_{\mathrm{C}}-k_{\mathrm{R}}\right)}{W_{0}} e^{2 i k_{\mathrm{C}}\left(z_{\mathrm{R}}-z_{j}\right)}+\frac{\left(k_{\mathrm{C}}-k_{\mathrm{L}}\right)\left(k_{\mathrm{C}}+k_{\mathrm{R}}\right)}{W_{0}} e^{i k_{\mathrm{C}}\left(z_{j}-z_{\mathrm{L}}\right)}\right] & \\ \frac{1}{i k_{\mathrm{R}}}\left\{1+\left[\frac{2 k_{\mathrm{R}}\left(k_{\mathrm{C}}+k_{\mathrm{L}}\right)}{W_{0}}+\frac{2 k_{\mathrm{R}}\left(k_{\mathrm{C}}-k_{\mathrm{L}}\right)}{W_{0}} e^{2 i k_{\mathrm{C}}\left(z_{\mathrm{R}}-z_{\mathrm{L}}\right)}-1\right] e^{2 i k_{\mathrm{R}}\left(z_{j}-z_{\mathrm{R}}\right)}\right\} & z_{\mathrm{R}}<z_{j} .\end{cases}
$$

As far as the wave number $k_{\mathrm{L}(\mathrm{C}, \mathrm{R})}$ is a real number [see Eqs. (14) and (15)], exponential terms seen in Eq. (18) are just complex numbers with the absolute value of 1 . On the other hand, when the wave number $k_{\mathrm{L}(\mathrm{C}, \mathrm{R})}$ is an imaginary number, these exponential terms are real numbers and are not more than 1. It should be emphasized that no exponential divergence occurs in any diagonal elements $D_{j}$. Even if the wave number $k_{\mathrm{C}}$ is a large imaginary number and the width of the rectangular reference potential $z_{\mathrm{R}}-z_{\mathrm{L}}$ is large, the denominator $W_{0}$ still has a finite value of $\left(k_{\mathrm{C}}+k_{\mathrm{L}}\right)\left(k_{\mathrm{C}}+k_{\mathrm{R}}\right)$, and therefore, division by zero or infinity never occurs in the practical computation of the regularized diagonal elements $D_{j}$. 
In the same manner, the ratios $R_{ \pm, j}$ are also given in analytical forms by

$$
R_{+, j}= \begin{cases}e^{i k_{\mathrm{L}} h} \frac{1+r_{+, 0} e^{2 i k_{\mathrm{L}}\left(z_{\mathrm{L}}-z_{j}\right)}}{1+r_{+, 0} e^{2 i k_{\mathrm{L}}\left(\mathrm{z}_{\mathrm{L}}-z_{j-1}\right)}} & z_{j}<z_{\mathrm{L}} \\ \frac{2 k_{\mathrm{L}}}{W_{0}} e^{i k_{\mathrm{L}}\left(z_{\mathrm{L}}-z_{j-1}\right)} e^{i k_{\mathrm{C}}\left(z_{j}-z_{\mathrm{L}}\right) \frac{\left(k_{\mathrm{C}}+k_{\mathrm{R}}\right)+\left(k_{\mathrm{C}}-k_{\mathrm{R}}\right) e^{2 i k_{\mathrm{C}}\left(z_{\mathrm{R}}-z_{j}\right)}}{1+r_{+, 0} e^{2 i k_{\mathrm{L}}\left(z_{\mathrm{L}}-z_{j-1}\right)}}} & z_{j-1}<z_{\mathrm{L}}<z_{j}<z_{\mathrm{R}} \\ e^{i k_{\mathrm{C}} h} \frac{\left(k_{\mathrm{C}}+k_{\mathrm{R}}\right)+\left(k_{\mathrm{C}}-k_{\mathrm{R}}\right) e^{2 i k_{\mathrm{C}}\left(z_{\mathrm{R}}-z_{j}\right)}}{\left(k_{\mathrm{C}}+k_{\mathrm{R}}\right)+\left(k_{\mathrm{C}}-k_{\mathrm{R}}\right) e^{2 i k_{\mathrm{C}}\left(z_{\mathrm{R}}-z_{j-1}\right)}} & z_{\mathrm{L}}<z_{j-1}, z_{j}<z_{\mathrm{R}} \\ \frac{2 k_{\mathrm{C}} e^{i k_{\mathrm{C}}\left(z_{\mathrm{R}}-z_{j-1}\right)} e^{i k_{\mathrm{R}}\left(z_{j}-z_{\mathrm{R}}\right)}}{\left(k_{\mathrm{C}}+k_{\mathrm{R}}\right)+\left(k_{\mathrm{C}}-k_{\mathrm{R}}\right) e^{2 i k_{\mathrm{C}}\left(z_{\mathrm{R}}-z_{j-1}\right)}} & z_{\mathrm{L}}<z_{j-1}<z_{\mathrm{R}}<z_{j} \\ e^{i k_{\mathrm{R}} h} & z_{\mathrm{R}}<z_{j-1}\end{cases}
$$

and

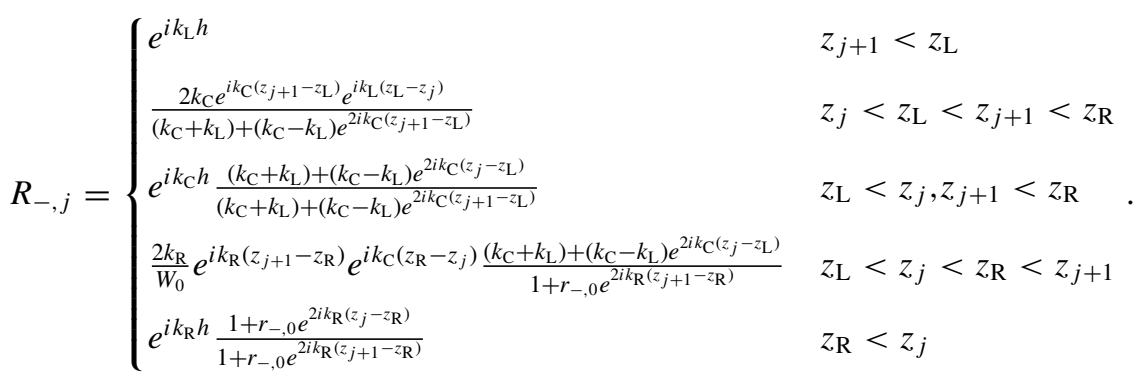

Here $h$ is real-space grid separation in the $z$ direction; $r_{ \pm, 0}$ are defined in the Appendix. One can easily see that these ratios $R_{ \pm, j}$ do not exponentially diverge, and their absolute values are less than 1 if the wave number $k_{\mathrm{L}(\mathrm{C}, \mathrm{R})}$ is a large imaginary number.

We thus obtain the regularization and ratio expression of the Green's function elements to avoid the numerical collapse discussed at the end of Sec. II A. Indeed, the numerical collapse never occurs in the improved Lippmann-Schwinger equation method in which the regularization and ratio expression are adopted because the Green's function components, $D_{j}$ and $R_{ \pm, j}$, are well defined without diverging, i.e., their absolute values are less than 1 if wave number $k_{\mathrm{L}(\mathrm{C}, \mathrm{R})}$ is a large imaginary number, as seen in Eqs. (18)-(20). Now we are ready to calculate electron transport through a long conductor between a pair of electrodes.

Here we briefly state the computational cost of the improved method. According to Eq. (2), the Green's function matrix operates on a vector obtained by the product of the potential difference $V_{\mathrm{dif}}^{(l)}(z)$ and the scattering wave function $\psi^{(l)}(z)$. The product of the Green's function matrix and the resultant vector still costs $\mathrm{O}(N)$, though the Green's function matrix is no longer in a separable form. This is easily understood as follows: Decomposing the Green's function matrix Eq. (17) into upper and lower triangular matrices, and a diagonal matrix, the product of each matrix and a vector is evaluated with computational costs of $\mathrm{O}(N)$. For details, see p. 189 in Ref. 21.

\section{PERFORMANCE TEST}

In this section, we present electron transport calculations of long conductor systems using both the improved and conventional Lippmann-Schwinger equation methods, and demonstrate the potential strength of the improved method discussed above. All of the calculations to be presented in this section are carried out as follows: Using an electronic structure calculation method based on the real-space finitedifference method, ${ }^{21,22}$ we calculate self-consistently the ground electronic state of a conductor system including jellium electrodes. The self-consistent potential and pseudopotential information ${ }^{23}$ thus determined is transmitted to the electron transport calculation codes based on the improved and conventional Lippmann-Schwinger equation methods. In the test we calculated scattering wave functions for the self-consistent potential obtained from the electronic structure calculations.

For a performance test, we employ long and simple systems with Al monoatomic linear chains. Figure 3 depicts a schematic representation of the $\mathrm{Al}$ chain systems, in which an atomic linear chain composed of $N \mathrm{Al}$ atoms is directly sandwiched between a pair of Al jellium electrodes. The Wigner-Seitz radius, which is the only parameter of the jellium model, is set to 2.0 bohrs, corresponding to the valence electron density of Al bulk. The number of atoms, $N$, changes to 3,8 , and 15 with the chain lengths of $10.8,37.8$, and 75.6 bohrs, respectively, in which the atom-atom distance is fixed to 5.4 bohrs. ${ }^{9,10,24}$ The distance between an end atom and the nearest electrode surface is also fixed at 2.6 bohrs. ${ }^{9,10,24}$ Therefore, the width of the rectangular reference potential $z_{\mathrm{R}}-z_{\mathrm{L}}$, which is one of the essential parameters concerning numerical collapse in the conventional Lippmann-Schwinger equation method, is measured as $16.0,43.0$, and 80.8 bohrs for $N=3,8$, and 15, respectively. The supercell dimension in the $x$ and $y$ directions

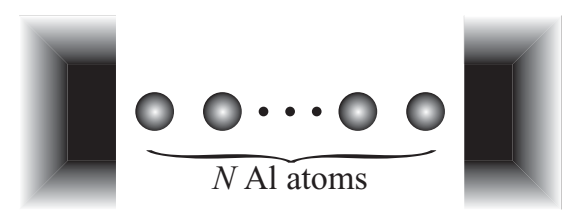

FIG. 3. A schematic representation of the Al chain systems used in the performance test. Spheres and black solid parts represent Al atoms and jellium parts, respectively. 
is $15.3 \times 15.3$ bohrs $^{2}$. The other important parameter $k_{\mathrm{C}}^{(l)}$ is evaluated using Eq. (14), which is associated with the height of the rectangular potential $0.48,0.47$, and 0.47 for $N=3,8$, and 15 , respectively. In the performance test, we calculate electron transmission under the assumption of the zero-bias limit. The two-dimensional cutoff energy of plane-wave expansion, $E_{\text {cut }}^{(2 \mathrm{D})}$, is taken to be $43.17 \mathrm{Ry}$. The real-space grid spacing in the $z$ direction is $\sim 0.47 \mathrm{bohr}$, which corresponds to a cutoff energy of $44.68 \mathrm{Ry}$ in the conventional plane wave method.

Using the conventional method for computing the Wronskian $W$ according to Eq. (12), we regard $\exp (t)$ as enough small that division by zero can occur, when $\Lambda=i k_{\mathrm{C}}^{(l)}\left(z_{\mathrm{R}}-z_{\mathrm{L}}\right)$ is real and smaller than a threshold value $\Lambda_{\text {cut }}$ (referred to as cutoff condition in the following). Thus, plane-wave expansion components of the Green's function satisfying the cutoff condition are excluded from the computation to complete calculations. This exclusion of information results in the reduction of two-dimensional cutoff energy of plane-wave expansion, $E_{\mathrm{cut}}^{(2 \mathrm{D})}$, because the wave number $k_{\mathrm{C}}^{(l)}$ is associated with the two-dimensional wave number vector $\boldsymbol{G}^{(l)}$, as seen in Eqs. (14) and (15).

Figures 4(a)-4(c) show electron transmissions as a function of energy of incident electrons for $N=3,8$, and 15 , respectively. One transmission curve (black solid) is obtained from the improved method, and the other two are obtained from the conventional method with the cutoff conditions $\Lambda_{\text {cut }}=-100$ and -200 . The cutoff condition $\Lambda_{\text {cut }}=-100$ is looser and introduces a lower two-dimensional cutoff energy $E_{\text {cut }}^{(2 D)}$ than $\Lambda_{\text {cut }}=-200$, as shown in Table I. In the case of $N=3$, the electron transmissions shown in Fig. 4(a) all behave the same, even for the looser cutoff condition of $\Lambda_{\text {cut }}=-100$. This is because the width of the rectangular reference potential is still small enough, and essential planewave expansion components of the Green's function are not excluded. Notably, these electron transmission curves are in good agreement with those reported in previous studies using a conventional Lippmann-Schwinger equation method ${ }^{9,10}$ and using the recursion transfer matrix method. ${ }^{25}$
TABLE I. Two-dimensional cutoff energy of plane-wave expansion, $E_{\text {cut }}^{(2 D)}$, taken into account in the performance test using the conventional method. Note that the cutoff energy is a function of the energy of incident electrons, $E$, as seen in Eq. (14).

\begin{tabular}{crrrrr}
\hline \hline & \multicolumn{2}{c}{$\Lambda_{\text {cut }}=-100$} & & \multicolumn{2}{c}{$\Lambda_{\text {cut }}=-200$} \\
\cline { 2 - 3 } \cline { 5 - 6 }$N$ & $E=-0.25$ & $E=0.0$ & & $E=-0.25$ & $E=0.0$ \\
\hline 3 & $38.49 \mathrm{Ry}$ & $38.99 \mathrm{Ry}$ & & $43.17 \mathrm{Ry}$ & $43.17 \mathrm{Ry}$ \\
8 & $4.85 \mathrm{Ry}$ & $5.35 \mathrm{Ry}$ & & $21.05 \mathrm{Ry}$ & $21.55 \mathrm{Ry}$ \\
15 & $0.97 \mathrm{Ry}$ & $1.47 \mathrm{Ry}$ & & $5.58 \mathrm{Ry}$ & $6.08 \mathrm{Ry}$ \\
\hline \hline
\end{tabular}

In the case of $N=8$ shown in Fig. 4(b), the electron transmission for the looser cutoff condition of $\Lambda_{\text {cut }}=-100$ exhibits different behavior than the others; e.g., the transmission curve of $\Lambda_{\text {cut }}=-100$ has the first onset at a higher energy than the two other curves that behave in the same way. This difference is caused by the exclusion of essential plane-wave expansion components of the Green's function, which leads to the limitation of the two-dimensional cutoff energy $E_{\text {cut }}^{(2 D)}$ as seen in Table I. The cutoff energies for $N=8$ and $\Lambda_{\text {cut }}=-100$ are not sufficient to describe the scattering wave functions of the $\mathrm{Al}$ chain system correctly. ${ }^{26}$ For the other case with the stricter cutoff condition of $\Lambda_{\text {cut }}=-200$, the electron transmission is coincident with that obtained by the improved method, because the computation for $\Lambda_{\text {cut }}=-200$ still takes into account the two-dimensional cutoff energy of the plane-wave expansion up to $21.05 \mathrm{Ry}$.

We now proceed to the model of the longest Al linear chain, in which $15 \mathrm{Al}$ atoms are in a line with a length of 75.6 bohrs. As seen in Fig. 4(c), the three transmission curves all exhibit different behavior. Focusing on the change in the transmission curves against the length of the $\mathrm{Al}$ chain, we can see that the first onset of the transmission curves obtained by the conventional method moves to the higher-energy side when the chain length is increased, while that obtained by the improved method retains its position. As mentioned in the previous paragraph, the shift of the onset position is associated with insufficiency of the two-dimensional cutoff
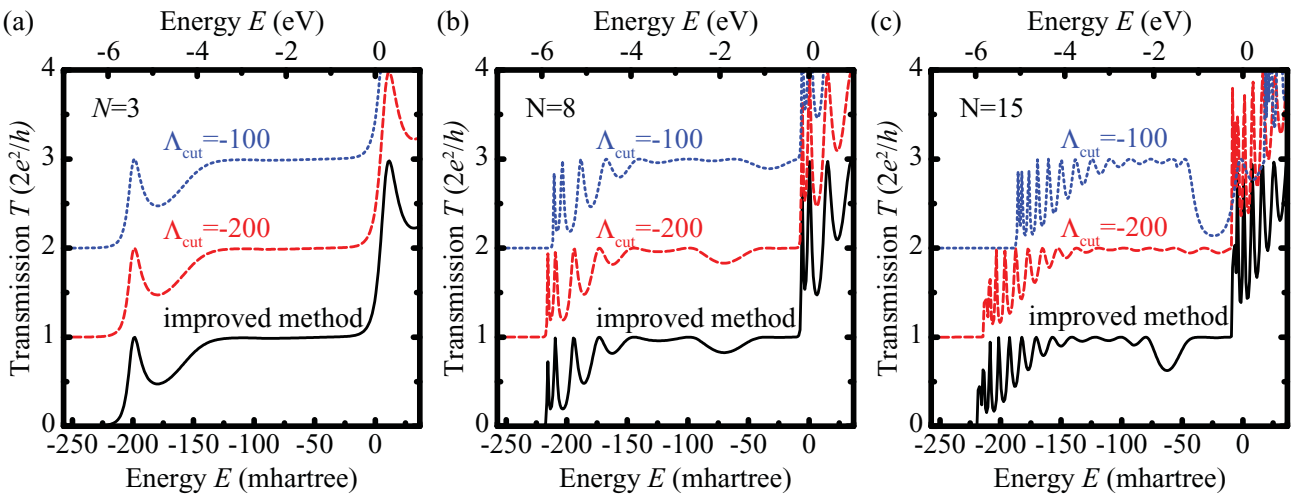

FIG. 4. (Color online) Electron transmissions of Al monoatomic linear chains as a function of energy of incident electrons. (a)-(c) show the electron transmissions for $N=3,8$, and 15, respectively. In each diagram the transmissions are calculated using the improved method (black solid curve) and using the conventional method with two different cutoff conditions of $\Lambda_{\text {cut }}=-200$ (red dashed curve) and -100 (blue dotted curve). For cutoff conditions, see main text. The transmission curves are vertically shifted for visibility. The energy is measured from the Fermi level. 
energy $E_{\text {cut }}^{(2 \mathrm{D})}$. Table I shows that not enough cutoff energy is taken into account for the two calculations using the conventional method. This suggests that for the longest Al chain system, the conventional method with not only the looser cutoff condition $\Lambda_{\text {cut }}=-100$ but also the stricter cutoff condition $\Lambda_{\text {cut }}=-200$ no longer works accurately due to the large separation between the electrodes. On the other hand, the Lippmann-Schwinger equation method that adopts the procedures of the regularization and ratio expression succeeds in calculating electron transmissions properly. In addition, the improved method is applicable to long conductor systems, for which the conventional Lippmann-Schwinger equation method has encountered numerical collapse.

\section{CONCLUSION}

To avoid numerical collapse in practical computation of electron transport property using the conventional LippmannSchwinger equation method, we introduced the procedures of the regularization and ratio expression of the retarded Green's function matrix elements. Due to this introduction, the computation of the Green's function no longer suffers from numerical collapse. Hence, large and long atomic and molecular structures sandwiched between a pair of electrodes can be treated to determine the electron-scattering wave functions using the improved Lippmann-Schwinger equation method. Employing long Al monoatomic linear chains up to 75.6 bohrs, we also demonstrated the numerical stability of the improved Lippmann-Schwinger equation method by adopting the regularization and ratio expression. Electron transport calculation under a finite bias-voltage application will be addressed in our future investigations.

\section{ACKNOWLEDGMENTS}

We are gratefully acknowledge Professor T. Ono of Osaka University for a number of comments and suggestions. This work is supported in part by the Strategic Japanese-German Cooperative Program of the Japan Science and Technology Agency and the German Research Foundation. Some of the computations were carried out by the supercomputer JuRoPa at Jülich Supercomputing Centre, Forschungszentrum Jülich.

\section{APPENDIX: ANALYTICAL SOLUTION OF EQ. (7) UNDER A RECTANGULAR POTENTIAL}

When a rectangular shape is selected as a one-dimensional reference potential $V_{\text {ref }}(z)$, as shown in Fig. 2, the pair of solutions of the differential equation Eq. (7), $y_{ \pm}(z)$, can be obtained in analytical form as

$$
y_{+}(z)= \begin{cases}e^{i k_{\mathrm{L}} z}+r_{+} e^{-i k_{\mathrm{L}} z} & z<z_{\mathrm{L}} \\ a_{+} e^{i k_{\mathrm{C}} z}+b_{+} e^{-i k_{\mathrm{C}} z} & z_{\mathrm{L}}<z<z_{\mathrm{R}} \\ t_{+} e^{i k_{\mathrm{R}} z} & z_{\mathrm{R}}<z\end{cases}
$$

and

$$
y_{-}(z)= \begin{cases}t_{-} e^{-i k_{\mathrm{L}} z} & z<z_{\mathrm{L}} \\ a_{-} e^{-i k_{\mathrm{C}} z}+b_{-} e^{i k_{\mathrm{C}} z} & z_{\mathrm{L}}<z<z_{\mathrm{R}}, \\ e^{-i k_{\mathrm{R}} z}+r_{-} e^{i k_{\mathrm{R}} z} & z_{\mathrm{R}}<z\end{cases}
$$

where $r_{ \pm}$and $t_{ \pm}$are reflection and transmission coefficients, respectively. Here $a_{ \pm}$and $b_{ \pm}$are coefficients of the propagating and backscattering waves or evanescent waves in the center region of the rectangular potential. Owing to the simple shape of the potential, these coefficients are all determined analytically as

$$
\begin{gathered}
r_{+}=\left[\frac{2 k_{\mathrm{L}}\left(k_{\mathrm{C}}+k_{\mathrm{R}}\right)}{W_{0}}+\frac{2 k_{\mathrm{L}}\left(k_{\mathrm{C}}-k_{\mathrm{R}}\right)}{W_{0}} e^{2 i k_{\mathrm{C}}\left(z_{\mathrm{R}}-z_{\mathrm{L}}\right)}-1\right] \\
\times e^{2 i k_{\mathrm{L}} z_{\mathrm{L}}} \equiv r_{+, 0} e^{2 i k_{\mathrm{L}} z_{\mathrm{L}}} \\
t_{+}=\frac{4 k_{\mathrm{L}} k_{\mathrm{C}}}{W_{0}} e^{i k_{\mathrm{L}} z_{\mathrm{L}}} e^{-i k_{\mathrm{R}} z_{\mathrm{R}}} e^{i k_{\mathrm{C}}\left(z_{\mathrm{R}}-z_{\mathrm{L}}\right)} \\
a_{+}=\frac{2 k_{\mathrm{L}}\left(k_{\mathrm{C}}+k_{\mathrm{R}}\right)}{W_{0}} e^{i k_{\mathrm{L}} z_{\mathrm{L}}} e^{-i k_{\mathrm{C}} z_{\mathrm{L}}} \\
b_{+}=\frac{2 k_{\mathrm{L}}\left(k_{\mathrm{C}}-k_{\mathrm{R}}\right)}{W_{0}} e^{i k_{\mathrm{L}} z_{\mathrm{L}}} e^{-i k_{\mathrm{C}} z_{\mathrm{L}}} e^{2 i k_{\mathrm{C}} z_{\mathrm{R}}}
\end{gathered}
$$

and

$$
\begin{gathered}
r_{-}=\left[\frac{2 k_{\mathrm{R}}\left(k_{\mathrm{C}}+k_{\mathrm{L}}\right)}{W_{0}}+\frac{2 k_{\mathrm{R}}\left(k_{\mathrm{C}}-k_{\mathrm{L}}\right)}{W_{0}} e^{2 i k_{\mathrm{C}}\left(z_{\mathrm{R}}-z_{\mathrm{L}}\right)}-1\right] \\
\times e^{-2 i k_{\mathrm{R}} z_{\mathrm{R}}} \equiv r_{-, 0} e^{-2 i k_{\mathrm{R}} z_{\mathrm{R}}} \\
t_{-}=\frac{4 k_{\mathrm{R}} k_{\mathrm{C}}}{W_{0}} e^{i k_{\mathrm{L}} z_{\mathrm{L}}} e^{-i k_{\mathrm{R}} z_{\mathrm{R}}} e^{i k_{\mathrm{C}}\left(z_{\mathrm{R}}-z_{\mathrm{L}}\right)} \\
a_{-}=\frac{2 k_{\mathrm{R}}\left(k_{\mathrm{C}}+k_{\mathrm{L}}\right)}{W_{0}} e^{-i k_{\mathrm{R}} z_{\mathrm{R}}} e^{i k_{\mathrm{C}} z_{\mathrm{R}}} \\
b_{-}=\frac{2 k_{\mathrm{R}}\left(k_{\mathrm{C}}-k_{\mathrm{L}}\right)}{W_{0}} e^{-i k_{\mathrm{R}} z_{\mathrm{R}}} e^{i k_{\mathrm{C}} z_{\mathrm{R}}} e^{-2 i k_{\mathrm{C}} z_{\mathrm{L}}}
\end{gathered}
$$

where $W_{0}$ is determined by Eq. (13), and $k_{\mathrm{L}}, k_{\mathrm{C}}$, and $k_{\mathrm{R}}$ defined as Eqs. (14) and (15) are the wave numbers in the $z$ direction in the left, center, and right regions of the reference potential, respectively.

\section{*s.tsukamoto@fz-juelich.de}

${ }^{1}$ M. Di Ventra, Electron Transport in Nanoscale Systems (Cambridge University Press, Cambridge, England 2008); Y. Imry and R. Landauer, Rev. Mod. Phys. 71, S306 (1999); S. Datta, in Electronic Transport in Mesoscopic Systems, Cambridge Studies in Semiconductor Physics and Microelectronic Engineering, Vol. 3, edited by H. Ahme, M. Pepper, and A. Broers (Cambridge University Press, Cambridge, 1995).
${ }^{2}$ K. S. Thygesen, M. V. Bollinger, and K. W. Jacobsen, Phys. Rev. B 67, 115404 (2003); M. Brandbyge, J.-L. Mozos, P. Ordejón, J. Taylor, and K. Stokbro, ibid. 65, 165401 (2002).

${ }^{3}$ M. Tsukada, K. Tagami, K. Hirose, and N. Kobayashi, J. Phys. Soc. Jpn. 74, 1079 (2005); K. Hirose, N. Kobayashi, and M. Tsukada, Phys. Rev. B 69, 245412 (2004); K. Hirose and M. Tsukada, ibid. 51, 5278 (1995). 
${ }^{4}$ Y. Egami, K. Hirose, and T. Ono, Phys. Rev. E 82, 056706 (2010); L. Kong, M. L. Tiago, and J. R. Chelikowsky, Phys. Rev. B 73, 195118 (2006); Y. Fujimoto and K. Hirose, ibid. 67, 195315 (2003). ${ }^{5}$ N. D. Lang, Phys. Rev. B 52, 5335 (1995).

${ }^{6}$ N. D. Lang, Phys. Rev. B 49, 2067 (1994).

${ }^{7}$ N. D. Lang and P. Avouris, Phys. Rev. Lett. 81, 3515 (1998).

${ }^{8}$ N. D. Lang and A. R. Williams, Phys. Rev. B 18, 616 (1978).

${ }^{9}$ Y. Asari, J. Nara, N. Kobayashi, and T. Ohno, Phys. Rev. B 72, 035459 (2005).

${ }^{10}$ N. Kobayashi, M. Aono, and M. Tsukada, Phys. Rev. B 64, 121402 (2001).

${ }^{11}$ S. Tsukamoto and K. Hirose, Phys. Rev. B 66, 161402 (2002).

${ }^{12}$ J. Nara, W. T. Geng, H. Kino, N. Kobayashi, and T. Ohno, J. Chem. Phys. 121, 6485 (2004).

${ }^{13}$ Y.-C. Chen and M. Di Ventra, Phys. Rev. B 67, 153304 (2003); M. Di Ventra and N. D. Lang, ibid. 65, 045402 (2001); N. D. Lang and P. Avouris, ibid. 64, 125323 (2001); M. Di Ventra, S. T. Pantelides, and N. D. Lang, Phys. Rev. Lett. 84, 979 (2000); S. Tsukamoto, M. Aono, and K. Hirose, Jpn. J. Appl. Phys. 41, 7491 (2002).

${ }^{14}$ C. Solterbeck, O. Tiedje, F. Starrost, and W. Schattke, J. Electron. Spectrosc. Relat. Phenom. 88-91, 563 (1998).

${ }^{15}$ This paper is aimed at calculating scattering wave functions of long conductor systems efficiently and precisely. For the purpose we exploit analytical solutions of a unperturbed reference system which comprises a pair of jellium electrodes. When treating the real-space Lippmann-Schwinger equation (1) directly, they may include more realistic electrodes and are free from the jellium approximation. However, they cannot utilize the analytical solutions anymore and computing cost increases drastically. This is not our present concern and is not examined in detail here.
${ }^{16}$ N. D. Lang, in Solid State Physics, edited by H. Ehrenreich, F. Seitz, and D. Turnbull, Vol. 28 (Academic Press, New York, 1973), pp. 225-300; N. D. Lang and W. Kohn, Phys. Rev. B 1, 4555 (1970); J. R. Smith, Phys. Rev. 181, 522 (1969).

${ }^{17} \mathrm{M}$. Thuthu and S. Fujino, in Computer Mathematics, Lecture Notes in Computer Science, Vol. 5081, edited by D. Kapur (Springer, Berlin, 2008), p. 108.

${ }^{18}$ For instance, in the case of a benzendithiol molecule suspended between a pair of jellium electrodes, the number of conjugate gradient iterations required up to convergence with a rectangular reference potential is on average about 30 times smaller than that with a constant reference potential.

${ }^{19}$ For a detailed estimate of the computational cost, see Ref. 12.

${ }^{20} \mathrm{At}$ a high-frequency component of the two-dimensional plane-wave expansion, it also remains possible that either of the wave numbers $k_{\mathrm{L}(\mathrm{R})}^{(l)}$ is a large imaginary number and the Wronskian $W^{(l)}$ diverges in an exponential manner.

${ }^{21} \mathrm{~K}$. Hirose, T. Ono, Y. Fujimoto, and S. Tsukamoto, First-Principles Calculations in Real-Space Formalism (Imperial College Press, London, 2005).

${ }^{22}$ T. Ono and K. Hirose, Phys. Rev. B 72, 085115 (2005); Phys. Rev. Lett. 82, 5016 (1999).

${ }^{23}$ N. Troullier and J. L. Martins, Phys. Rev. B 43, 1993 (1991); K. Kobayashi, Comput. Mater. Sci. 14, 72 (1999).

${ }^{24}$ K. Hirose, N. Kobayashi, and M. Tsukada, Thin Solid Films 464465, 255 (2004).

${ }^{25}$ N. Kobayashi, M. Brandbyge, and M. Tsukada, Surf. Sci. 433-435, 854 (1999).

${ }^{26}$ Our preliminary calculation revealed that this Al linear chain system requires the two-dimensional cutoff energy of at least 10.0 Ry. 